\title{
Ultra-High Nonlinear Lead Silicate Photonic Crystal Fiber with Two Zero Dispersion for Supercontinuum Generation Applications
}

\section{Pradeep Kumar Gupta}

NMIMS School of Pharmacy and Technology Management

\section{Binay Prakash Akhouri}

Suraj Group of Institutions

\section{Abhishek Kumar}

Mody University of Science and Technology

Manoj Mishra

Mody University of Science and Technology

\section{Brajraj Singh}

Mody University of Science and Technology

Mohit Sharma ( $\square$ mohitsharmac@gmail.com )

Mody University of Science and Technology https://orcid.org/0000-0002-9835-2260

\section{Research Article}

Keywords: photonic crystal fiber (PCF), PCF, SC generation, nonlinear coefficient

Posted Date: September 20th, 2021

DOl: https://doi.org/10.21203/rs.3.rs-705463/v1

License: (9) (i) This work is licensed under a Creative Commons Attribution 4.0 International License. Read Full License 


\title{
Ultra-High Nonlinear Lead Silicate Photonic Crystal Fiber with Two Zero Dispersion for Supercontinuum Generation Applications
}

\author{
Pradeep Kumar Gupta ${ }^{1}$, Binay Prakash Akhouri², Abhishek Kumar ${ }^{3}$, Manoj Mishra ${ }^{3}$, Brajraj Singh ${ }^{3}$ and Mohit Sharma ${ }^{3, *}$ \\ ${ }^{1}$ Department of Applied Sciences and Humanities SVKMS' NMIMS Deemed University, Shirpur Maharashtra, India-425405 \\ ${ }^{2}$ Department of Physics, Suraj Singh Memorial College, Ranchi Jharkhand, India -834008 \\ ${ }^{3}$ Department of Physics, School of Liberal Arts and Science, Mody University of Science and Technology, Lakshmangarh, Sikar, Rajasthan, \\ India-835215 \\ *Corresponding Author : mohitsharmac@gmail.com
}

\begin{abstract}
This paper presents an unique type of Lead silicate (SF57) photonic crystal fiber (PCF) with eleven rings of air holes. The PCF promises to yield very large nonlinearity $\sim 13169 \mathrm{~W}^{-1} \mathrm{~km}^{-1}$ at $0.75 \mu \mathrm{m}$, $\sim 8607 \mathrm{~W}^{-1} \mathrm{~km}^{-1}$ at $1.064 \mu \mathrm{m}$ and $\sim 4640 \mathrm{~W}^{-1} \mathrm{~km}^{-1}$ at $1.55 \mu \mathrm{m}$. The PCF has two zero dispersion at wavelength $0.3 \mu \mathrm{m}$ and $1.50 \mu \mathrm{m}$. The value of nonlinearities is highest reported till now. The PCF contains very low optical mode field, causes large nonlinearity which makes it best suitable PCF for generating supercontinuum.
\end{abstract}

\section{Introduction}

Photonic crystal fiber (PCF) was first discovered by knight et. al. in 1996 [1] and generally have the defected central core surrounded by air holes cladding in a regular triangular or hexagonal lattice pattern. The effective refractive index contrast between core and cladding with flexibility of design promises excellent optical properties like high nonlinear coefficient [2,3], zero dispersion [2,3], endless single mode propagation [4], and high birefringence [4] etc. PCFs can achieve a high effective nonlinear coefficient by keeping effective mode field area as small as possible. The chromatic dispersion of the PCFs can be tuned by tailoring the air holes pitch and air holes cladding arrangement. These properties are the best suitable for a variety of novel application including optical soliton parametric amplification and supercontinuum generation [5-8]. The high value of nonlinear coefficient can be achieved by tight mode confinement with the use of glasses with greater intrinsic material nonlinearity coefficients than silica. Lead 
Silicate PCFs, especially SF57 glass PCF has a high nonlinear refractive index $\left(4.1 \times 10^{-19} \mathrm{~m}^{2} / W\right)$, transmission up to wavelength $3.5 \mu \mathrm{m}$, good thermal and crystallization stability [3] as compared to the fused silica glass PCF, where fused silica has a nonlinear refractive index $\left(2.1 \times 10^{-20} \mathrm{~m}^{2} / W\right)$ and transmission limit upto wavelength $2 \mu \mathrm{m}$.

In a PCF large nonlinearity can be realized by making a very tight confinement of the optical mode in the core region, which could be achieved either by decreasing the core area with some modification in the cladding design or increasing the refractive index contrast between core and cladding by introducing soft glass materials with large nonlinear coefficient. Due to large nonlinear coefficient of non-silica materials, several authors have investigated PCFs which are made of these materials. For example, PCFs made of fluoride glass, bismuth oxide, lead silicate, tellurite and chalcogenide etc., exhibit large effective nonlinearity which is suitable for devices that rely on optical nonlinearity. Particular interest has been shown to lead silicate glass with nonlinear coefficient which is 20 times larger than that of silica glass and has good thermal and crystallization stability $[13,14]$. Lead silicate glass fibers exhibit attenuation $\sim 1-3$ $\mathrm{dB} / \mathrm{m}$ at the operating wavelength $1.55 \mu \mathrm{m}$, whereas chalco-sulfide glass has fiber attenuation $3-4 \mathrm{~dB} / \mathrm{m}$ at $1.55 \mu \mathrm{m}[14]$ and it has higher thermal expansion coefficient than silica, which may allow more flexibility in the fabrication process. A brief comparison of thermal, crystalline and other properties of soft glasses have been discussed by Feng et al. [14]. PCFs with large effective optical nonlinearity require very low threshold power to generate broadband supercontinuum, which make them attractive for application in supercontinuum (SC) generation.

Due to large effective nonlinearity, PCFs made of SF57 glass have remain very attractive nonlinear medium for SC generation. Extensive investigations have been carried out towards evolving designs of SF57 glass PCFs to achieve large effective nonlinearity and small dispersion so as to get flat broadband supercontinuum in these fibers. Along this direction, several authors have studied effective nonlinear coefficient of SF57 glass PCFs. For example, effective nonlinear coefficient of 112, 500 and $1860 \mathrm{~W}^{-1} \mathrm{~km}^{-1}$ at $1.55 \mu \mathrm{m}$ operating wavelength were respectively reported by Xing-Ping et al. [9], Tiwari et al. [10] and Leong et al. [12]. Several efforts have been made to achieve wideband SC spectra in SF57 glass PCFs [912]. For example, Xing-Ping et al. [9] generated SC spectra from $1300 \mathrm{~nm}$ to $1900 \mathrm{~nm}$, Leong et al. [12] were able to generate SC spectra spanning more than $1000 \mathrm{~nm}$ by using $300 \mathrm{fs}$ pulses in a $6.8 \mathrm{~cm}$ long fiber. Miret et al. [11] have reported $3 \mathrm{~dB}$ flat SC spectra spanning over $\sim 1500 \mathrm{~nm}$ by using femtosecond pulse in a $15 \mathrm{~cm}$ PCF which exhibits normal dispersion. Buczynski et al. [13] have also reported about 
$1500 \mathrm{~nm}$ wide SC spectra in lead-bismuth-gallate glass PCF by pumping femtosecond pulses at operating wavelength $1540 \mathrm{~nm}$. Tiwari et al. [10] generated SC spectra from $1000 \mathrm{~nm}$ to $3200 \mathrm{~nm}$ by using $50 \mathrm{fs}$ pump pulse of peak power $2 \mathrm{~kW}$ in a $15 \mathrm{~cm}$ long PCF. Along this direction, we focus our attention to achieve broadband SC generation at low input power. The objective of the present study is to two-fold. First, to design a SF57 glass PCF which exhibits large nonlinearity and low dispersion and then we use this fiber to achieve broadband SC spectra employing pump pulses with low peak power. The proposed fiber can be fabricated without many difficulties since several authors have demonstrated the fabrication of PCFs with soft glass. For example, Leong et al. [12] have fabricated a soft glass PCF with core diameter $0.95 \mu \mathrm{m}$, Xing-Ping et al. [9] have studied SF57 PCF with small air hole of diameter $0.5 \mu \mathrm{m}$ which was fabricated by Institute of Photonics and Advance Sensing at University of Adelaide, Australia. Buczynski et al. [13] have also demonstrated the fabrication of lead silicate PCF with core diameter $3.36 \mu \mathrm{m}$ by using extrusion technique [15].

The organization of the paper is as follows: In section 2, we have described the necessary computational procedure. In section 3, we have described the PCFs. Important results have been discussed in this section. A brief conclusion has been added in section 4 .

\section{Theoretical Model}

Fiber dispersion is one of the most important parameters relevant to supercontinuum generation. The total dispersion $D_{C}(\lambda)$ of the fiber is the sum of waveguide dispersion and material dispersion i.e., $D_{C}(\lambda)=D_{M}(\lambda)+D_{W}(\lambda)$ and numerically expressed by $D_{C}(\lambda)=-\frac{\lambda}{c} \frac{d^{2} n_{e f f}}{d \lambda^{2}}$, where $\lambda$ is operating wavelength, $c$ is the speed of light, $n_{e f f}=\frac{\lambda}{2 \pi} \beta$ is effective refractive index of the fiber, $n$ is the refractive index of SF57 glass and $\beta$ is the propagation constant. The Sellmeier's equation $\mathrm{n}^{2}(\lambda)=1+\sum_{i} \frac{B_{i} \lambda^{2}}{\lambda^{2}-C_{i}}$ has been utilized to evaluate material dispersion [16], where $B_{i}$ and $C_{i}$ is the Sellmeier's coefficients of the material. For SF57 glass $B_{1}=1.8165127, \quad B_{2}=0.42889364, \quad B_{3}=1.07186278, \quad \lambda_{1}=$ $0.0143704198 \mu \mathrm{m}, C_{2}=0.0592801172 \mu \mathrm{m}$ and $C_{3}=121.419942 \mu \mathrm{m}$. The effective nonlinear coefficient $(\gamma)$ of the fiber can be calculated using $\gamma=\left(2 \pi n_{2} / \lambda A_{\text {eff }}\right) \times 10^{3} \mathrm{~W}^{-1} \mathrm{~km}^{-1}$, where $n_{2}=$ $4.1 \times 10^{-19} \mathrm{~m}^{2} / \mathrm{W}$ and $A_{e f f}$ is an effective mode area of the fiber, which is defined [3-10] by $\mathrm{A}_{\text {eff }}=$ $\frac{\left(\iint_{-\infty}^{\infty}|E|^{2} \mathrm{dxdy}\right)^{2}}{\iint_{-\infty}^{\infty}|\mathrm{E}|^{4} \mathrm{dxdy}}$. The single modeness of a PCF is characterized by the normalized V parameter whose 
effective value $\quad V_{\text {eff }}$ can be expressed as $V_{\text {eff }}=2 \pi \frac{\Lambda}{\lambda} \sqrt{n_{\text {core }}^{2}-n_{\text {eff }}^{2}}$, where $\Lambda$ is the hole pitch, $n_{\text {core }}$ is the refractive index of the core. The single mode cut-off for photonic crystal fiber is $V_{\text {eff }} \leq$ 4.1 [17]. The confinement loss is calculated by using finite element method (FEM) [18, 19], which is numerically represented as $C L=8.686 k_{0} \operatorname{Im}\left(n_{e f f}\right)$, where $\operatorname{Im}\left(n_{e f f}\right)$ is the imaginary part of the effective refractive index and $k_{0}=2 \pi / \lambda$ is the wavenumber in free space [20].

\section{Result and discussion}

With the aim of achieving high nonlinearity with two zero dispersion. We designed a unique type of body centered hexagonal PCF by confining its optical mode field as small as possible to achieve high nonlinearity with two zero dispersion, whereas the simple fiber design was unable to confine the optical mode field small as compared to the proposed design with desired two zero dispersion wavelengths. We therefore modify the design by additional introduction of small air holes in each hexagonal lattice layer of air holes. This modification leads a very tight confinement of optical mode field in the core region, which helps to achieve large nonlinearity (Fig. 1(a)).

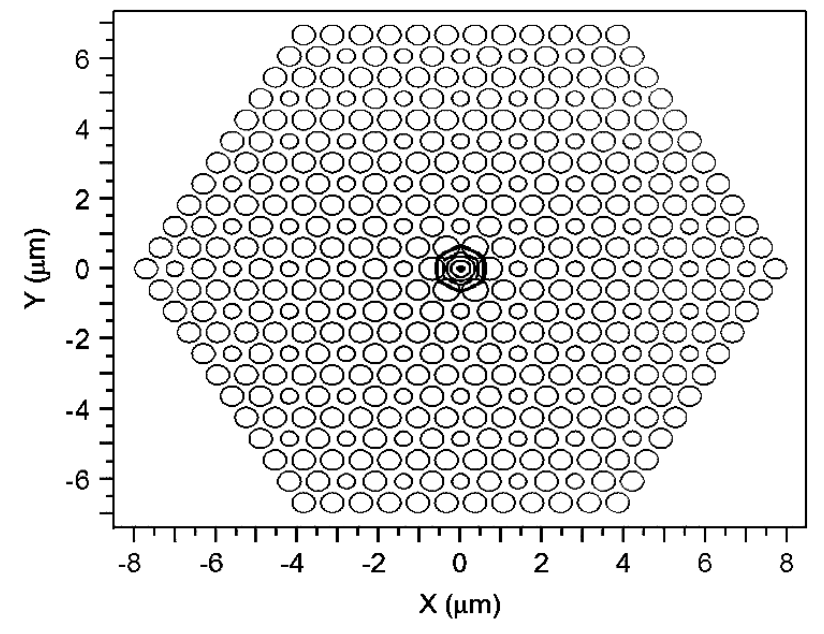

(a)

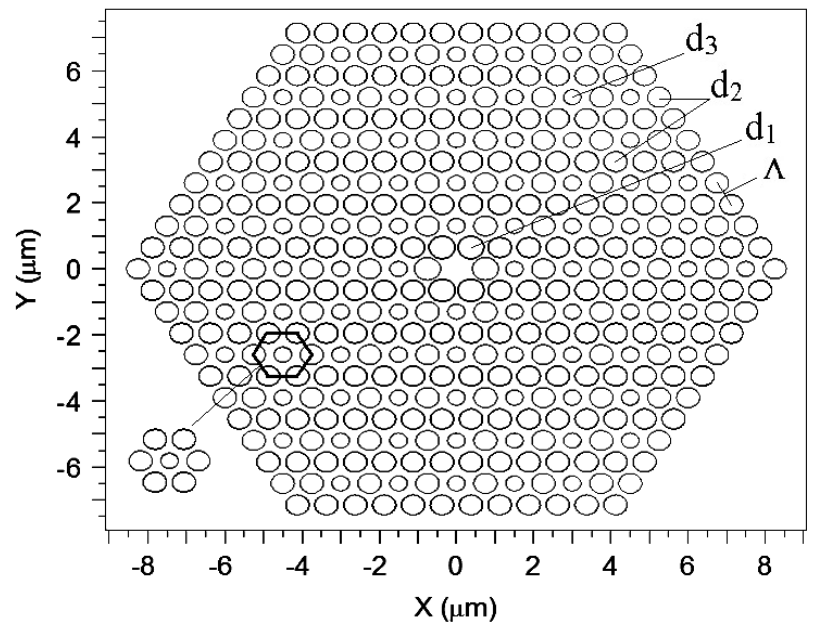

(b)

Fig.1 (a) Transverse mode profile of proposed Fiber, $(b)$ Contour map of transverse index profile at $\mathrm{Z}=0$ and where $\mathrm{d}_{1}=0.9 \times \Lambda \mu \mathrm{m}, \mathrm{d}_{2}=0.8 \times \Lambda \mu \mathrm{m}, \mathrm{d}_{3}=0.6 \times \Lambda \mu \mathrm{m}$. In caption figure of body centered hexagonal ring design. 


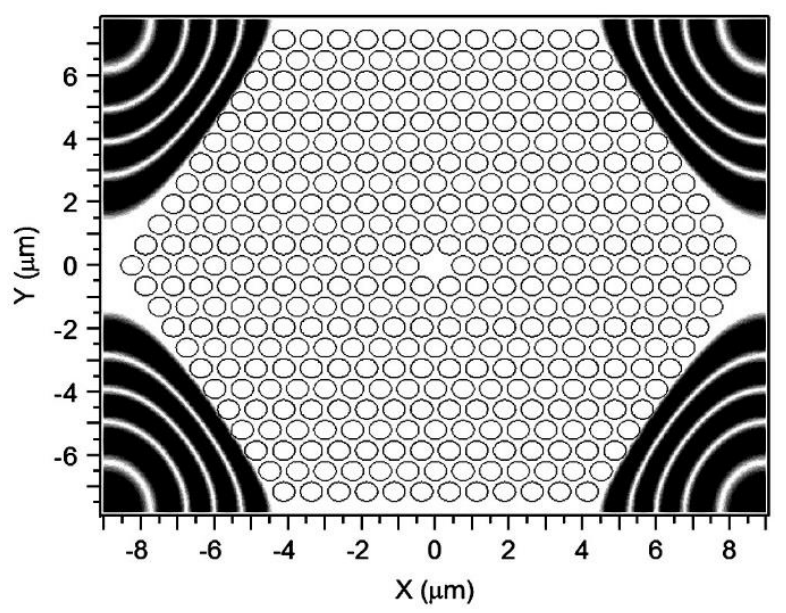

Fig.2 Leakages of optical mode from simple fiber with $d=0.9 \times \Lambda \mu \mathrm{m}$.

The schematic of the fiber is shown in Fig. 1(b), which has eleven rings of circular air holes with unique geometry, where a small air hole of diameters $\mathrm{d}_{3}=0.6 \times \Lambda \mu \mathrm{m}$ is surrounded by the large air holes with $\mathrm{d}_{2}=0.8 \times \Lambda \mu \mathrm{m}$ in a periodic hexagonal pattern. The air hole diameter $\mathrm{d}_{1}$ of the first ring $\left(\mathrm{d}_{1}=0.9 \times \Lambda \mu \mathrm{m}\right)$ is slightly larger than the neighboring air hole which ensures small effective area and outermost ring $\mathrm{d}_{2}=0.8 \times \Lambda \mu \mathrm{m}$. Fig.2 illustrate the leakage of the optical mode from simple design of PCF with $d=0.9 \times \Lambda \mu \mathrm{m}$. It may be observed that the PCF is not able to confine the optical mode inside the core. Hance, start leaking from the cladding. After several alteration, the optical mode is able to remain in the core of the fiber, which may be seen from Fig.1(a).

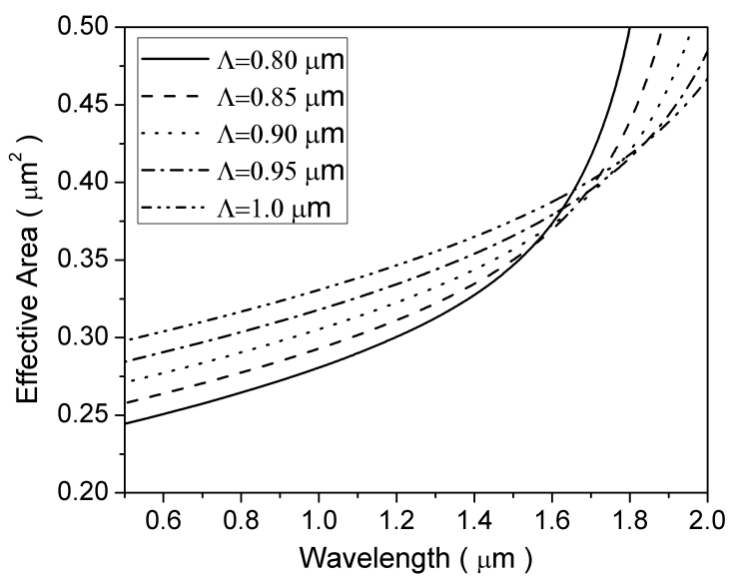

Fig. 3 Effective area at different hole pitch with $d_{1}=0.9 \times \Lambda \mu m, d_{2}=0.8 \times \Lambda \mu m, d_{3}=0.6 \times \Lambda \mu m$. 


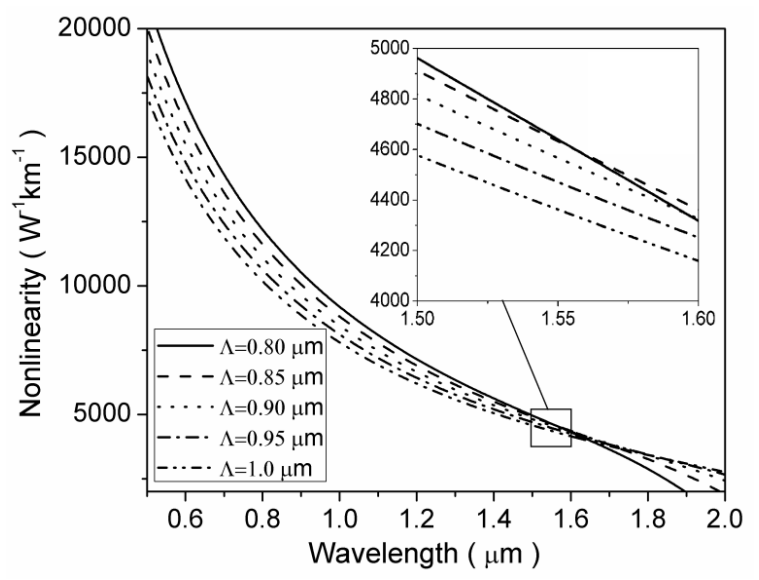

Fig. 4 Effective Nonlinear Coefficient at different hole pitch with $d_{1}=0.9 \times \Lambda \mu m, d_{2}=0.8 \times \Lambda \mu m$, $d_{3}=0.6 \times \Lambda \mu m$.

We have investigated the effective nonlinear coefficient $(\gamma)$ of the proposed fiber. Fig. 4 shows the nonlinearity of the fundamental mode for five different values of hole pitch $\Lambda$ (i.e., $\Lambda=0.8 \mu \mathrm{m}, \Lambda=$ $0.85 \mu \mathrm{m}, \Lambda=0.9 \mu \mathrm{m}, \Lambda=0.95 \mu \mathrm{m}$ and $\Lambda=1 \mu \mathrm{m}$ ). Due to small effective area of the fiber core (Fig. 3), PCFs shows very high nonlinear coefficient in the visible range and starts decreasing the nonlinearity with increasing the hole pitch $\Lambda$. Effective mode area of the PCFs can decrease up to certain point beyond that point PCFs start increasing effective area which leads the decrease in nonlinearity as in Fig. 4. The large effective nonlinearity noticed $\sim 4640 \mathrm{~W}^{-1} \mathrm{~km}^{-1}$ with effective area $0.3583 \mu \mathrm{m}^{2}$ at the wavelength $1.55 \mu \mathrm{m}$. The effective area gradually increases at higher wavelengths, which cause of getting high nonlinearity at hole pitch $\Lambda=0.8 \mu \mathrm{m}$ (Fig. 3). Table.1 depicts the large nonlinearities with effective area at variation of different pump wavelength for supercontinuum generation.

Table.1. Nonlinearities with effective area at variation of different wavelength.

\begin{tabular}{|c|c|c|c|c|c|c|}
\hline \multirow{2}{*}{$\begin{array}{l}\text { Period } \\
\Lambda(\boldsymbol{\mu m})\end{array}$} & \multicolumn{2}{|c|}{$0.75 \mu m$} & \multicolumn{2}{|c|}{$1.064 \mu m$} & \multicolumn{2}{|c|}{$1.55 \mu m$} \\
\hline & $\gamma\left(\mathbf{W}^{-1} \mathbf{k m}^{-1}\right)$ & $A_{e f f}\left(\mu m^{2}\right)$ & $\gamma\left(W^{-1} \mathbf{k m}^{-1}\right)$ & $A_{e f f}\left(\mu m^{2}\right)$ & $\gamma\left(\mathbf{W}^{-1} \mathbf{k m}^{-1}\right)$ & $A_{e f f}\left(\mu m^{2}\right)$ \\
\hline 0.80 & 13169 & 0.26094 & 8607 & 0.32186 & 4640 & 0.35836 \\
\hline 0.85 & 12543 & 0.27395 & 8261 & 0.28518 & 4633 & 0.35894 \\
\hline 0.90 & 11971 & 0.28705 & 7934 & 0.29713 & 4565 & 0.36423 \\
\hline 0.95 & 11446 & 0.30021 & 7626 & 0.30938 & 4470 & 0.37202 \\
\hline 1.00 & 10964 & 0.31342 & 7335 & 0.33451 & 4361 & 0.3813 \\
\hline
\end{tabular}




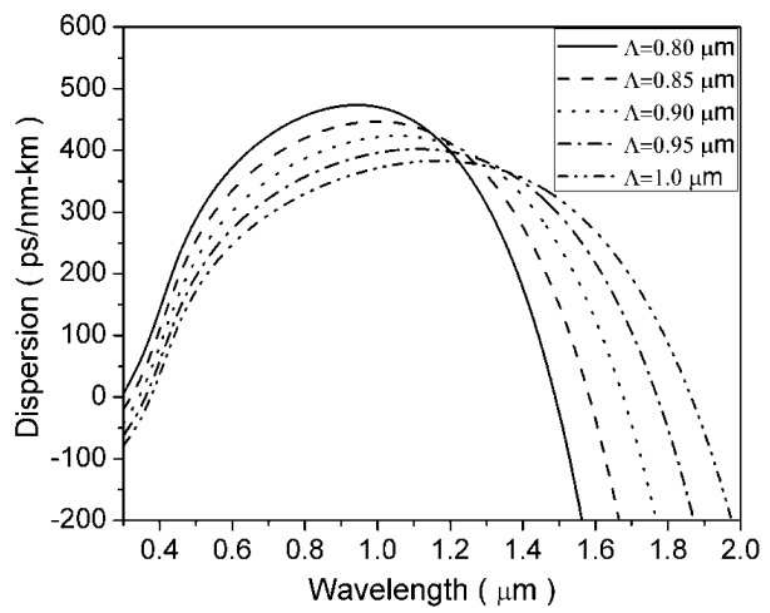

Fig. 5 Dispersion Profile of the fiber at $d_{1}=0.9 \times \Lambda \mu m, d_{2}=0.8 \times \Lambda \mu m, d_{3}=0.6 \times \Lambda \mu m$.

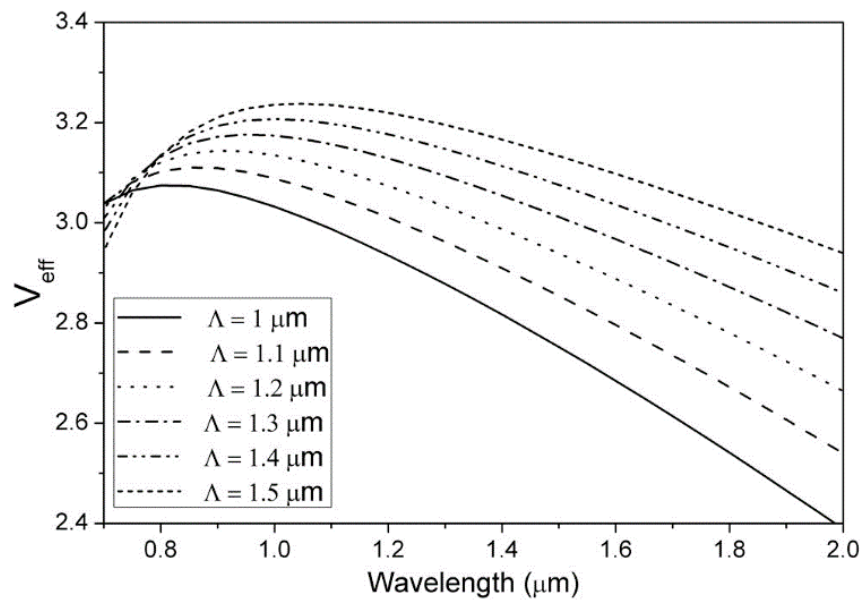

(a)

(b)

Fig. $6 \mathrm{~V}$ parameter of the fiber at $d_{1}=0.9 \times \Lambda \mu \mathrm{m}, d_{2}=0.8 \times \Lambda \mu \mathrm{m}, d_{3}=0.6 \times \Lambda \mu \mathrm{m}$.

We have calculated the dispersion properties of the proposed fiber. Fig. 5 shows the variation of dispersion of the fundamental mode for five different values of hole pitch (i.e., $\Lambda=0.8 \mu \mathrm{m}, \Lambda=0.85 \mu \mathrm{m}$ $, \Lambda=0.9 \mu \mathrm{m}, \Lambda=0.95 \mu \mathrm{m}$ and $\Lambda=1 \mu \mathrm{m})$. Due to large refractive index differences between core and cladding, PCFs shows the two zero dispersion at the wavelength $0.3 \mu \mathrm{m}$ and $1.50 \mu \mathrm{m}$ at $\Lambda=$ $0.8 \mu \mathrm{m}$ which may be tuned by varying the air hole pitch. The second zero dispersion wavelength shifts towards higher wavelength as increasing the air hole pitch $\Lambda$. Hence, the desired chromatic dispersion can be achieved by changing air hole pitch $\Lambda$. As the generation of wide supercontinuum needs the pulse pumped in an anomalous regime with low dispersion then $\mathrm{PCF}$ with $\Lambda=0.85 \mu \mathrm{m}$ may be a better choice 
for pump wavelength at $1.55 \mu \mathrm{m}$, as it promises the zero dispersion at wavelengths $\sim 0.35 \mu \mathrm{m}$ and $\sim 1.57 \mu \mathrm{m}$. Whereas, from Fig 6, it is amply clear that the proposed PCF is single moded over a wide range of wavelengths.

\section{Conclusion}

We have designed a highly nonlinear photonic crystal fiber, which contains eleven rings of air holes. These air holes are arranged in a body centered hexagonal lattice pattern. The diameter of the air holes in the innermost ring is the largest while that of the outermost ring is smallest. By optimizing the structure parameters, the nonlinear coefficient value is achievable up to $\sim 4640 \mathrm{~W}^{-1} \mathrm{~km}^{-1}$ at $1.55 \mu \mathrm{m}$ and the two zero dispersion wavelength is at $0.3 \mu \mathrm{m}$ and $1.55 \mu \mathrm{m}$. Finite difference time domain method has been adopted to study the optical fiber dispersion and non-linear characteristics of the fiber. This proposed highly nonlinear PCFs is easy to fabricate by using flam brushing technique. With regard to the two zero dispersion wavelength, highly nonlinearity PCFs are a good candidate to the supercontinuum generation at wavelength $1.55 \mu \mathrm{m}$.

\section{References}

[1] Knight, J. C., T. A. Birks, P. St J. Russell, and D. M. Atkin. "All-silica single-mode optical fiber with photonic crystal cladding." Optics letters 21, no. 19 (1996): 1547-1549.

[2] Akhouri, Binay P., and Pradeep K. Gupta. "Waveguiding effect on optical spatial solitons in centrosymmetric photorefractive materials." Journal of Optics 46, no. 3 (2017): 281-286.

[3] Sharma, Mohit, Swapan Konar, and Kaisar R. Khan. "Supercontinuum generation in highly nonlinear hexagonal photonic crystal fiber at very low power." Journal of Nanophotonics 9, no. 1 (2015): 093073.

[4] Bhattacharya, Rakhi, and S. Konar. "Extremely large birefringence and shifting of zero dispersion wavelength of photonic crystal fibers." Optics \& Laser Technology 44, no. 7 (2012): 2210-2216.

[5] Sharma, M., Borogohain, N. and Konar, S., 2013. Index guiding photonic crystal fibers with large birefringence and walk-off. Journal of lightwave technology, 31(21), pp.3339-3344. 
[6] Mishra, Manoj, Mohit Sharma, and Prachi Gupta. "Compact MIM plasmonic ring resonator for nano-interconnect applications." Physica E: Low-dimensional Systems and Nanostructures 130 (2021): 114711.

[7] Akhouri, B. P., and P. K. Gupta. "Potential formalism of optical spatial soliton propagation in a two-photon photovoltaic-photorefractive material under open circuit condition." Indian Journal of Physics 88, no. 4 (2014): 421-425.

[8] The dynamics of screening spatial solitons in photorefractive-photovoltaic crystals incorporating higher order space charge fields. Journal of optoelectronics and advanced materials 16(3-4), 463$470,2014$.

[9] Akhouri, Binay Prakash, and P. K. Gupta. "The dynamics of screening spatial solitons in photorefractive-photovoltaic crystals incorporating higher order space charge fields." Journal of Optoelectronics and Advanced Materials 16, no. 3-4 (2014): 463-470.'

[10] Tiwari, Manish, and Vijay Janyani. "Two-Octave Spanning Supercontinuum in a Soft Glass Photonic Crystal Fiber Suitable for 1.55\$ $1 \mathrm{mu}$ \$ m Pumping." Journal of lightwave technology 29, no. 23 (2011): 3560-3565.

[11] Miret, Juan Jose, E. Silvestre, and P. Andrés. "Octave-spanning ultraflat supercontinuum with softglass photonic crystal fibers." Optics express 17, no. 11 (2009): 9197-9203.

[12] Leong, Julie YY, Periklis Petropoulos, Jonathan HV Price, Heike Ebendorff-Heidepriem, S. Asimakis, R. C. Moore, K. E. Frampton et al. "High-nonlinearity dispersion-shifted lead-silicate holey fibers for efficient $1-\mu \mathrm{m}$ pumped supercontinuum generation." Journal of Lightwave Technology 24, no. 1 (2006): 183.

[13] Buczynski, R., H. T. Bookey, D. Pysz, R. Stepien, I. Kujawa, J. E. McCarthy, A. J. Waddie, A. K. Kar, and M. R. Taghizadeh. "Supercontinuum generation up to $2.5 \mu \mathrm{m}$ in photonic crystal fiber made of lead-bismuth-galate glass." Laser Physics Letters 7, no. 9 (2010): 666.

[14] Feng, Xian, Arshad K. Mairaj, Daniel W. Hewak, and Tanya M. Monro. "Nonsilica glasses for holey fibers." Journal of lightwave technology 23, no. 6 (2005): 2046. 
[15] Monro, T. M., Kiang, K. M., Lee, J. H., Frampton, K., Yusoff, Z., Moore, R., \& Richardson, D. J. High nonlinearity extruded single-mode holey optical fibers. In Optical Fiber Communication Conference (p. FA1). Optical Society of America. (2002, March).

[16] Mogilevtsev, Dmitri, T. A. Birks, and P. St J. Russell. "Localized function method for modeling defect modes in 2-D photonic crystals." Journal of lightwave technology 17, no. 11 (1999): 2078.

[17] Sharma, Mohit, and S. Konar. "Three octave spanning supercontinuum by red-shifted dispersive wave in photonic crystal fibers." Journal of Modern Optics 63, no. 5 (2016): 501-510.

[18] Khan, Kaisar R., and Thomas X. Wu. "Short pulse propagation in wavelength selective indexguided photonic crystal fiber coupler." IEEE Journal of Selected Topics in Quantum Electronics 14, no. 3 (2008): 752-757.

[19] Khan, Kaisar R., Mohammad F. Mahmood, and Anjan Biswas. "Coherent super continuum generation in photonic crystal fibers at visible and near infrared wavelengths." IEEE Journal of Selected Topics in Quantum Electronics 20, no. 5 (2014): 573-581.

[20] Gong, Tao-rong, Feng-ping Yan, Lin Wang, Pen Liu, Yi-fang Li, and Shui-sheng Jian. "A kind of photonic crystal fiber with high birefringence and low cofinement loss." Optoelectronics Letters 4, no. 2 (2008): 110-113. 